\title{
Professional conferences in the COVID-19 era
}

\author{
Seunggon Jung, DDS \\ Section Editor of JKAOMS \\ Department of Oral and Maxillofacial Surgery, School of Dentistry, Chonnam National University, Gwangju, Korea
}

The COVID-19 pandemic continues in 2021. Vaccination has begun, but it is difficult to predict the end of the pandemic. Face-to-face contact is discouraged to prevent the spread of highly virulent COVID-19, and physical distancing is affecting entire societies including education systems and industry. In January 2020, the annual oral cancer reconstruction workshop held by Korean Association of Oral and Maxillofacial Surgeons (KAOMS) and Korean Association of Maxillofacial Plastic and Reconstructive Surgeons (KAMPRS) was canceled because of COVID-19. The universities postponed the start of spring semester and conducted online lectures. As a result, many professors had to depend unfamiliar online lecture system and learning management system (LMS). In early 2020, it was expected that the COVID-19 pandemic would slow in the summer with increasing temperatures. This was a reasonable prediction based on the pattern of SARS-CoV-1, which started in November 2002 and lasted until July 2003. However, the season did not affect the COVID-19 pandemic caused by SARS-CoV-2. Many academic associations, including KAOMS, chose to temporarily postpone their annual conferences, and many subsequently decided to hold them online. Some institutions, including European Association for Cranio-Maxillo-Facial Surgery and Asian Association of Oral and Maxillofacial Surgeons cancelled their congresses for the year. However, most autumn conferences, including that of the KAMPRS, were held online. Development of online education has been steadily increasing with the spread of the

\section{Seunggon Jung}

Department of Oral and Maxillofacial Surgery, School of Dentistry, Chonnam National University, 33 Yongbong-ro, Buk-gu, Gwangju 61186, Korea

TEL: +82-62-530-5863

E-mail: seunggon.jung@jnu.ac.kr

ORCID: https://orcid.org/0000-0003-2008-3205

(c) This is an open-access article distributed under the terms of the Creative Commons Attribution Non-Commercial License (http://creativecommons.org/ licenses/by-nc/4.0/), which permits unrestricted non-commercial use, distribution, and reproduction in any medium, provided the original work is properly cited. Copyright (C) 2021 The Korean Association of Oral and Maxillofacial Surgeons. All rights reserved.
Internet and was vastly employed in the COVID-19 era. Such online professional conferences would not have been possible in the early 2000s before the advent of smartphones, video calls, and online lecture platforms.

In traditional academic conferences, participants gather at a hotel or convention center. Academic conferences are central for exchange and dissemination of knowledge. They are also platforms to form important professional networks. Participants are inspired and motivated by oral presentations, poster presentations, symposia, and lectures.

In online conferences, pre-recorded lectures are streamed as on-demand video via an asynchronous platform (e.g., Classum) or according to a typical conferences schedule via a synchronous platform (e.g., Zoom). Some conferences held real time sessions to allow interaction between the speaker and participants.

Online conferences have many advantages both from the perspective of the conference organizer and that of conference participants. Conference organizers can reduce indirect costs and costs of venue and catering although there will be expenses for the on-line lecture system ${ }^{1}$. Also, costs of flights, transport, and accommodation for invited speakers can be eliminated. From the perspective of the conference participants, accessibility increases, and entry barriers are lowered since it is possible to attend the international conference without the costs of flight and accommodation. And participants do not need to vacate their hospital. Because conventional conferences usually provided sessions in parallel, participants often had to choose to attend one session over the other ${ }^{1}$. In online conferences, participants can attend every session. Ondemand video lectures can be paused and replayed, offering opportunity to repeat the lecture for better understanding. In addition, reduction of organizing costs can result in lower registration fees. An average $42 \%$ decrease in registration fee was observed during the COVID-19 pandemic, although not all groups chose this option ${ }^{2}$.

Time zone differences can be problematic and act as a 
loco-regional limitation in online conferences on a synchronous platform. And some characteristics may work as disadvantages. Some speakers may be uncomfortable with online lectures, as they can be recorded without permission. Participants attending online conference in their room must deal with numerous distractions. Exchange and dissemination of knowledge, the original role of a conference, is difficult to ensure when online. Karkar-Esperat ${ }^{3}$, in his study of student experiences in online classes, reported that the students faced challenges with English language proficiency, isolation, instructor's lack of experience, and lack of motivation. Also, valuable networking and discussion opportunities are lost with online participation ${ }^{4}$.

Shebansky ${ }^{5}$ reported that technology adoption in language education is primarily hampered by time constraints and lack of technical support, and many educators will not adopt a new technology until they have evidence of its value. Likewise, the conferences wouldn't have been held online unless it had been special situation of COVID-19. However, the sustainability of online conferences is controversial. Some expect they will decrease in popularity, and some think they will continue along with traditional conferences ${ }^{1,2,4}$.

Although the future of the COVID-19 pandemic is uncertain, hybrid conference can be held after reaching community immunity. In hybrid conference, domestic participant can attend the conference in face to face and oversea participants can attend the conference remotely. Therefore, consideration is needed to ensure face-to-face interactions while not losing quality of education and academic exchanges via online for hybrid conference. The pandemic provides a good opportu- nity to incorporate technological advances into conferences. Although online conferences are unfamiliar and have some disadvantages, they could aid in the education and networking of oral and maxillofacial surgeons upon active participation of colleagues even after the pandemic.

\section{Conflict of Interest}

No potential conflict of interest relevant to this article was reported.

\section{References}

1. Zargaran D, Zargaran A, Phillips G, Theofanis AP, Atkins J. COVID-19: a unique opportunity to upgrade medical conferences. J Plast Reconstr Aesthet Surg 2020. https://doi.org/10.1016/ j.bjps.2020.08.135 [Epub ahead of print]

2. Mubin O, Alnajjar F, Shamail A, Shahid S, Simoff S. The new norm: computer science conferences respond to COVID-19. Scientometrics 2020. https://doi.org/10.1007/s11192-020-03788-9 [Epub ahead of print]

3. Karkar-Esperat TM. International graduate students' challenges and learning experiences in online classes. J Int Stud 2018;8:172235. https://doi.org/10.5281/zenodo.1468076

4. Speirs V. Reflections on the upsurge of virtual cancer conferences during the COVID-19 pandemic. Br J Cancer 2020;123:698-9. https://doi.org/10.1038/s41416-020-1000-x

5. Shebansky WJ. Blended learning adoption in an ESL context: obstacles and guidelines. TESL Can J 2018;35:52-77. https://doi. org/10.18806/tesl.v35i1.1284

How to cite this article: Jung S. Professional conferences in the COVID-19 era. J Korean Assoc Oral Maxillofac Surg 2021;47:12. https://doi.org/10.5125/jkaoms.2021.47.1.1 